\title{
SISTEMA DE AQUISIÇÃO DE DADOS PARA EQUIPAMENTO DE MEDIDA DA PERMEABILIDADE INTRÍNSECA DO SOLO AO AR ${ }^{(1)}$
}

\author{
Luciano Roberto da Silveira ${ }^{(2)}$, Alexsandro dos Santos Brito ${ }^{(3)}$, Jaedson Cláudio \\ Anunciato Mota ${ }^{(3)}$, Sergio Oliveira Moraes ${ }^{(4)} \&$ Paulo Leonel Libardi ${ }^{(4)}$
}

\begin{abstract}
RESUMO
A permeabilidade intrínseca - ou simplesmente permeabilidade do solo ao ar - é uma propriedade importante para a identificação de alterações no espaço poroso do solo causadas pelas práticas de manejo, na estimativa de propriedades do solo mais difíceis e onerosas e na composição de modelos de fluxo de fluidos em solos agrícolas e em solos contaminados. O objetivo do presente estudo foi construir um sistema de aquisição de dados (módulo eletrônico e programa computacional) para a medida da permeabilidade do solo ao ar em laboratório, utilizando-se materiais disponíveis no local e ferramentas computacionais de acesso livre. $\mathrm{O}$ sistema de aquisição de dados mostrou-se bastante preciso na determinação da permeabilidade do solo ao ar, com intervalo de confiança de $9,42 \pm 0,085 \mu^{2}(95 \%)$, para uma amostra-padrão constituída de partículas com diâmetro de 0,106 a $0,250 \mathrm{~mm}$ da fração areia de um Latossolo Vermelho-Amarelo textura média. A estimativa da permeabilidade do solo ao ar, considerando a viscosidade dinâmica do ar em função da temperatura, foi significativamente maior que a estimativa com valor fixo de viscosidade dinâmica em aproximadamente $20^{\circ} \mathrm{C}$. A medição realizada em uma amostra de solo com estrutura indeformada de um Latossolo Vermelho foi tão precisa quanto à da amostra-padrão, e a estimativa da massa de água removida da amostra foi de $3,27 \mathrm{mg}$.
\end{abstract}

Termos de indexação: transdutor de pressão, sensores, microcontrolador.

\footnotetext{
(1) Parte da Tese de Doutorado do segundo autor, a ser apresentada ao Programa de Pós-Graduação em Solos e Nutrição de Plantas da Escola Superior de Agricultura "Luiz de Queiroz" - ESALQ/USP. Recebido para publicação em maio de 2010 e aprovado em dezembro de 2010.

${ }^{(2)}$ Mestrando em Agronomia - Física do Ambiente Agrícola, Escola Superior de Agricultura "Luiz de Queiroz" - ESALQ/USP. Caixa Postal 9, Av. Pádua Dias 11, CEP 13418-900 Piracicaba (SP). E-mail: lroberto@esalq.usp.br (bolsista da Capes).

(3) Doutorandos em Agronomia - Solos e Nutrição de Plantas, ESALQ/USP. E-mail: alexsb@esalq.usp.br (bolsista da Capes), jcamota@esalq.usp.br (bolsista do CNPq).

(4) Professor do Departamento de Engenharia de Biossistemas, ESALQ/USP. E-mail: somoraes@esalq.usp.br, pllibard@esalq.usp.br
} 


\title{
SUMMARY: DATA ACQUISITION SYSTEM FOR MEASUREMENT EQUIPMENT OF SOIL AIR PERMEABILITY
}

\begin{abstract}
The intrinsic permeability or soil air permeability is an important soil property for the identification of changes in soil porous space caused by soil tillage, in estimations of soil properties that are expensive and difficult to be determined in the laboratory, and for the formulation of flux models in cropped and contaminated soils. The purpose of this study was to develop a data acquisition system (electronic module and software) for intrinsic permeability measurement, in laboratory. High precision of the acquisition system was observed in the estimation of this property, with a confidence interval of $9.42 \pm 0.085 \mu^{2}$ (significant at the $5 \%$ level), in a standard soil sample consisting of particles with diameter from 0.25 to $0.106 \mathrm{~mm}$ of the sand fraction of a medium texture Yellow Red Latosol. The estimate of soil air intrinsic permeability considering the air dynamic viscosity as a function of temperature was significantly greater than the estimate with fixed air dynamic viscosity at approximately $20^{\circ} \mathrm{C}$. The measurement precision of air permeability in an undisturbed Red Latosol sample was high and similar to that of the standard sample and the estimate of water mass removed from the soil sample (3.27 $\mathrm{mg})$.
\end{abstract}

Index terms: pressure transducer, sensors and microcontroller.

\section{INTRODUÇÃO}

A importância da quantificação da permeabilidade do solo ao ar deve-se, por exemplo, à necessidade de caracterizar o espaço poroso e identificar mudanças na estrutura do solo, ocasionadas pelas práticas de manejo (Blackwell et al., 1990; Cavalieri et al., 2009); e estabelecer correlações com outras propriedades do solo que são de difícil determinação, como é o caso da condutividade hidráulica (Loll et al., 1999), e com a extração de vapor de contaminantes encontrados nesse meio poroso (Fahran et al., 2001).

O primeiro aparato para medição da permeabilidade do solo ao ar foi proposto por Kirkham (1946) a partir da equação de Darcy-Buckingham, medindo o fluxo de ar por meio de uma amostra de solo submetida a um baixo gradiente de pressão de ar, decrescente com o tempo, servindo para utilização tanto no campo como em laboratório. Em termos do equipamento, as pesquisas dos últimos anos buscaram aprimorar o método, principalmente na automação da aquisição de dados, tornando o processo de medição mais fácil e preciso. Iversen et al. (2001) construíram um equipamento baseado principalmente no desenvolvido por Steinbrenner (1959) e Fish \& Koppi (1994), adicionando um regulador de pressão e três fluxímetros. Esse aparato permite a medição em campo ou laboratório (amostras de 100 e $3.140 \mathrm{~cm}^{3}$ ), utilizando um manômetro de água.

Mckenzie \& Dexter (1996) também desenvolveram um permeâmetro baseado no modelo de Kirkham (1946), adaptado para medida da permeabilidade ao ar em agregados. Neves et al. (2004) o aperfeiçoaram, adicionando um sistema de aquisição de dados, com um transdutor de pressão diferencial (850S - TP1 da Vectus, com faixa de medida de 0 a $10.000 \mathrm{~Pa}$ ) e programa computacional AqDXY 3.0 (Lyns Tecnologia). Recentemente, Silva et al. (2009), utilizando dois permeâmetros, um com sistema automático de aquisição de dados (Neves et al., 2004) e outro convencional, com medidas de tempo empregando um cronômetro e medidas de pressão com manômetro dotado de visor de cristal líquido, verificaram que a permeabilidade do solo ao ar mostrou-se sensível às alterações da densidade e do conteúdo de água no solo e que há alta correlação da permeabilidade com o parâmetro de ajuste alpha do modelo de van Genuchten (1980) e com a porosidade de aeração de amostras equilibradas na tensão de $10 \mathrm{kPa}$.

Há outros equipamentos que usam o método desenvolvido por Kirkham (1946) e aqueles que utilizam gradiente de pressão de ar constante. Tyner et al. (2005) desenvolveram um equipamento mais sofisticado, no qual o ar bombeado continuamente (fluxo contínuo) passava por uma câmara de desumidificação e medição da umidade relativa $(\mathrm{HIH}$, Honeywell) e vazão (FMA3100, Omega), antes de entrar em contato com a amostra de solo (pequena coluna). Ao longo da coluna de solo eram instalados cinco transdutores de pressão diferencial (PX170, Omega); após o ar deixar a amostra, a umidade relativa era medida novamente. Dessa forma, estes autores verificaram que a umidade relativa do ar antes e depois de passar pelo solo foi se igualando gradativamente, ao longo das $44 \mathrm{~h}$ de análise. A remoção de água ocasionou queda nas pressões medidas em todos os pontos da coluna de solo, sendo mais acentuada na última porção da coluna, por manter umidade alta durante a maior parte do processo. Nesse equipamento, os dados foram armazenados em um datalogger (21X, Campbell Scientific Inc., Logan, UT). 
Diante dos diversos modelos de permeâmetro encontrados na literatura, este trabalho objetivou construir um sistema de aquisição de dados que possibilitasse a medida da permeabilidade intrínseca do solo ao ar em amostras indeformadas de solo e o monitoramento de eventuais mudanças no conteúdo de água da amostra, adaptado a um permeâmetro de ar com gradiente de pressão decrescente de ar, com câmara de desumidificação.

\section{MATERIAL E MÉTODOS}

\section{Confecção do sistema automático de aquisi- ção de dados}

O sistema de aquisição de dados, composto do módulo eletrônico e do programa computacional (PermeAR v.1.0), foi construído nos Laboratórios de Eletrônica e de Física do Solo do Departamento de Engenharia de Biossistemas da Escola Superior de Agricultura "Luiz de Queiroz" - ESALQ/USP.

No módulo eletrônico foram utilizados dois sensores de temperatura de silício (modelo LM35, National Semiconductor); dois sensores de umidade relativa capacitivos (modelo HIH-4000, Honeywell) e um transdutor de pressão diferencial (modelo MPXV5004DP, Freescale) com faixa de operação de 0 a $3,92 \mathrm{kPa}$, sensibilidade de $1,0 \mathrm{Mv} \mathrm{Pa}^{-1}$ e acurácia de $\pm 1,5 \%$ da voltagem de fundo de escala. Todos os sensores foram interligados a um PC, por intermédio de um microcontrolador embarcado (modelo Basic Step M8, Tato ind.), que possui conversor A/D interno de 10 bits (oito canais) e referência de voltagem interna.

O programa computacional (PermeAR v.1.0) foi desenvolvido com a utilização de ferramentas de acesso livre, como o compilador Delphi 7 Personal (Borland 2002), a biblioteca de comunicação serial ComPort v.3.10 (Crnila, 2005), a biblioteca para gráficos Sgraph v.2.4 (Pod'yachev, 2001), além de ícones gratuitos e outros que foram criados.

\section{Permeabilidade do solo ao ar e monitoramento das modificações durante o processo}

Para determinação da permeabilidade do solo ao ar, o sistema de aquisição foi instalado em cilindro de ar, usado para armazenamento de GLP - gás de cozinha (Neves et al., 2004), com volume de $0,03135 \mathrm{~m}^{3}$, ligado a uma câmara de desumidificação, composta de sílica-gel, com entrada para injeção de ar, a partir de um compressor de ar manual. O objetivo da câmara de desumidificação é homogeneizar o ar quanto à umidade, no momento da determinação da permeabilidade em amostras de solo, nos diferentes tempos.

Entre a câmara de desumidificação e o cilindro de ar existe uma tubulação com uma válvula para liberação do ar para o cilindro e uma válvula de ajuste fino, necessária para equilíbrio da pressão inicial no processo de determinação da permeabilidade. $\mathrm{O}$ suporte para amostra indeformada de solo localiza-se na parte superior do cilindro de ar (Mckenzie \& Dexter, 1996; Neves et al., 2004), ligado ao cilindro por uma tubulação com válvula, que permite a passagem do ar do cilindro para a amostra de solo.

No interior do cilindro de ar foi instalado um par de sensores de temperatura e de umidade relativa. Outro par foi instalado em uma câmara, acima da amostra de solo, que se comunica com a atmosfera por um pequeno orifício. Com isso, monitora-se a condição inicial do ar dentro do cilindro e após ter passado pela amostra de solo. O transdutor de pressão foi instalado no circuito eletrônico e conectado ao cilindro de ar por uma mangueira pneumática (detalhes do aparelho na Figura 1).

O método (gradiente de pressão decrescente) para determinação da permeabilidade foi desenvolvido por Kirkham (1946), sendo a queda do gradiente de pressão em função do tempo dada pela equação 1:

$$
\log P_{a 1}-\log P_{a 2}=\frac{k_{a} A P_{a t m}}{2,3 L \eta V}\left(t_{2}-t_{1}\right)
$$

em que $P_{a 1}$ e $P_{a 2}$ são as pressões manométricas $(\mathrm{Pa})$ nos tempos (s) $t_{1}$ (inicial) e $t_{2}$ (final), respectivamente; $k_{a}$, a permeabilidade do solo ao ar $\left(\mathrm{m}^{2}\right) ; A$, a área da seção transversal da amostra de solo; $P_{a t m}$, a pressão atmosférica local $(\mathrm{Pa}) ; L$, a altura do anel volumétrico utilizado $(\mathrm{m}) ; \eta$, a viscosidade dinâmica do ar $(\mathrm{Pa} \mathrm{s})$; e $V$, o volume do cilindro de $\operatorname{ar}\left(\mathrm{m}^{3}\right)$.

Denominando $k_{a} A P_{a t m} / 2,3 L \eta V$ de $S$ (inclinação), uma vez que esse termo representa o coeficiente angular da regressão linear da pressão em função do tempo, tem-se (equação 2):

$$
\log P_{a 2}=-S t+\log P_{a 1}
$$

Conhecendo o coeficiente angular (S), pode-se calcular $\mathrm{k}_{\mathrm{a}}$ :

$$
k_{a}=\frac{2,3 L \eta V}{A P_{a t m}} \times|S|
$$

A pressão de ar para a determinação da permeabilidade é muito baixa em comparação a $P_{a t m}$ e a temperatura é a ambiente, o que permite tratar o ar no cilindro como um gás ideal. Unindo a esse fato a possibilidade de calcular a pressão de saturação do vapor d'água $-e_{s}-$ (antes e após passar pela amostra de solo), utilizando as medidas de temperatura na equação de Tétens (equação 4 , temperatura - t em ${ }^{\circ} \mathrm{C}$ ) e a pressão parcial absoluta do vapor d'água $\left(e_{a}\right.$, antes e após o ar passar pela amostra de solo), empregando a medida de umidade relativa $(U R)$ e a $e_{s}$ (equação 5), pode-se calcular a massa de vapor d'água $\left(m_{v}\right)$ contida no ar antes e após a passagem pela amostra de solo (equação 6). 
(a)

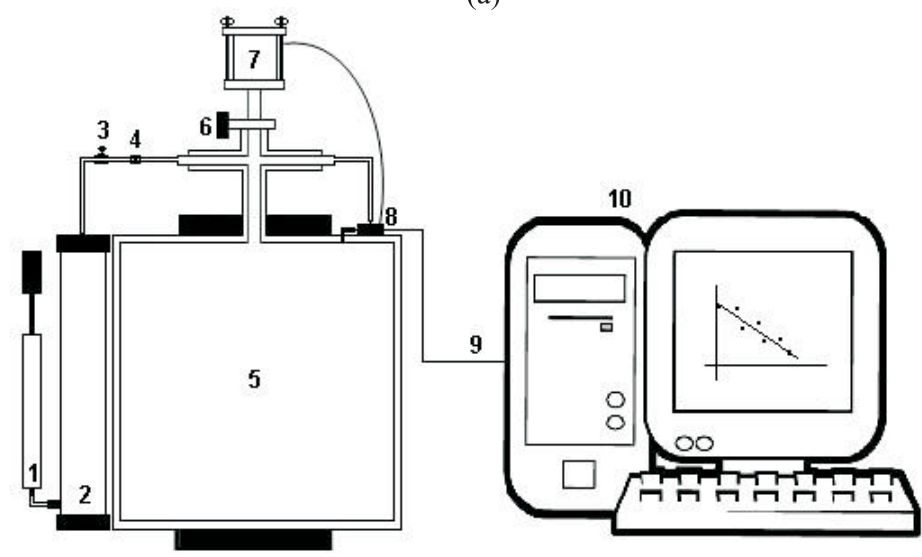

(b)

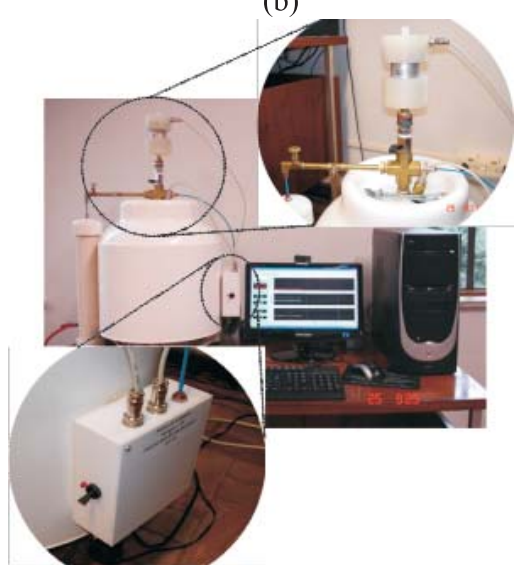

Figura 1. Diagrama esquemático (a) constituído por: 1: bomba de ar; 2: cilindro de desumidificação do ar preenchido com sílica-gel; 3: válvula de controle de fluxo; 4: válvula de escape para ajuste fino da pressão interna; 5: cilindro de ar; 6: válvula de controle de fluxo; 7: compartimento de alocação da amostra indeformada de solo; 8: sistema de aquisição de dados constituído por transdutor de pressão e sensores de temperatura e umidade; 9: cabo de transmissão de dados (DB 9); 10: microcomputador para processamento dos dados; e fotografia do equipamento para medida da permeabilidade intrínseca do solo ao ar (b).

$$
\begin{array}{r}
e_{s}=610,8 P a \times \exp ^{\frac{17,3 \times t}{237,3+t}} \\
e_{a}=\left(U R \times e_{s}\right) / 100 \\
m_{v}=\left(\bar{M}_{H_{2} O} \times e_{a} / R \times T\right) \times V_{a r}
\end{array}
$$

em que $\bar{M}_{\mathrm{H}_{2} \mathrm{O}}$ é a massa molar da água $=18 \mathrm{~g} \mathrm{~mol}^{-1}$; $R$, a constante universal dos gases ideais $=$ 8,314 $\mathrm{Pa} \mathrm{m}^{3} \mathrm{~mol}^{-1} \mathrm{~K}^{-1}$; $T$, a temperatura em Kelvin; e $V_{a r}$, o volume parcial de ar que passa pela amostra de solo. Assim, durante o processo de queda da pressão (Pa) haverá uma massa ou um volume de ar $\left(V_{a r}\right)$ proporcional, que, passando pela amostra de solo, removerá certa massa de água $\left(m_{r}\right)$, o que possibilita descrever o processo pela seguinte equação:

$$
m_{r}=\frac{\bar{M}_{v} \times V}{R T} \int_{e_{a}}^{e_{a}^{\prime}} d e_{a}
$$

em que $e_{a}$ e $e_{a}$ são as pressões parciais do vapor d'água do ar antes e após passar pela amostra de solo, respectivamente.

Para verificar o erro do equipamento na determinação da permeabilidade do solo ao ar, foi confeccionada uma amostra-padrão, utilizando um anel volumétrico (com diâmetro e altura de $0,05 \mathrm{~m}$ ) preenchido com a fração areia (diâmetro de partículas entre 0,250 e 0,106 mm) de um Latossolo Vermelho Amarelo textura média e com as extremidades delimitadas por uma tela silk screen (120 fios). Uma amostra indeformada coletada de um Latossolo Vermelho e equilibrada a $0,4 \mathrm{~m}$ de tensão em funil de Haines foi também utilizada para verificar o funcionamento do sistema de aquisição de dados.
Com a amostra-padrão, procedeu-se à análise da influência da viscosidade do ar em função da temperatura, utilizando os sensores de temperatura, e considerou-se a permeabilidade com viscosidade fixa e com viscosidade em função da temperatura como tratamentos (40 repetições), sendo testados pelo teste F e teste de média $t$.

\section{RESULTADOS E DISCUSSÃO}

\section{Avaliação do sistema de aquisição de dados}

O programa computacional PermeAR v.1.0 foi desenvolvido com as seguintes funções: estabelecer a comunicação serial entre o computador (PC) e o microcontrolador (cabo serial DB9); obter a leitura dos sensores; converter os valores digitais provindos do microcontrolador em unidades de temperatura $\left({ }^{\circ} \mathrm{C}\right)$, umidade relativa (\%) e pressão $(\mathrm{Pa})$; e exibir medidas instantâneas de forma numérica no monitor e acumular as medidas em duas interfaces: uma gráfica e outra no formato de planilha (Figura 2). Em ambas as interfaces o usuário tem a possibilidade de monitorar o processo de medição em tempo real das variáveis estudadas. O PermeAR v.1.0 permite, ainda, configurar o intervalo de tempo de varredura (0,1 a $5,0 \mathrm{~s})$ para monitoramento das variáveis no início do processo de medição e o intervalo de tempo de amostragem $(0,5$ a $5,0 \mathrm{~s})$; selecionar a porta de comunicação com o módulo eletrônico; zerar a pressão manométrica de fundo de escala, para corrigir eventuais variações; visualizar os gráficos no todo ou em detalhes; além de possuir uma interface com o programa Microsoft Excel, na qual o usuário tem a possibilidade de transferir todos os dados e gráficos, selecionando a faixa de interesse. 
Uma vez que tanto os sensores como o microcontrolador exigem voltagem de entrada de 5 volts, utilizou-se um transformador estabilizado para 9 volts $(500 \mathrm{~mA})$ e um regulador de voltagem KA7805TO220 (FairChild Semiconductor ${ }^{\circledR}$ ) que suporta voltagem e corrente de entrada de até 35 volts e $1 \mathrm{~A}$, respectivamente, e regula a voltagem de saída em 5 volts. O sinal dos sensores de temperatura passa por um amplificador operacional CA3140 (Intersil $®$ ) para se tornar compatível com a resolução do conversor A/D. O esquema completo do circuito eletrônico é mostrado na figura 3 .

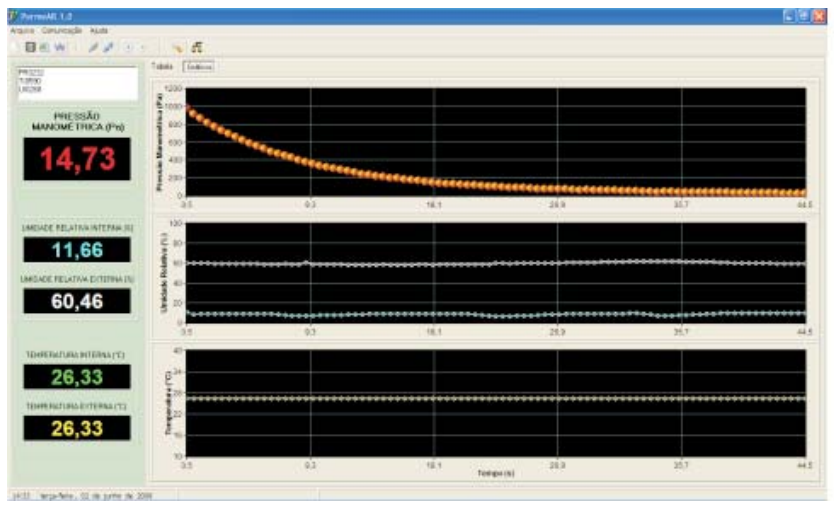

Teste com amostra-padrão de solo confeccionada e uma única amostra de Latossolo Vermelho indeformada

Os testes mostraram que o sistema de aquisição de dados funcionou, em seu conjunto, como esperado, uma vez que as exigências para o perfeito funcionamento dos componentes eletrônicos foram atendidas (Figura 4). Para a amostra-padrão, o intervalo de confiança da permeabilidade ao ar, a $95 \%$, foi de $9,25 \pm 0,087 \mu \mathrm{m}^{2}$ (40 repetições), com valor fixo de viscosidade dinâmica do ar. Estimando a viscosidade do

Figura 2. Janelas principais do programa computacional PermeAR v.1.0 com mostradores instantâneos da pressão manométrica, temperaturas e umidades relativas, interna e externa, e gráficos obtidos durante o processo de medição.

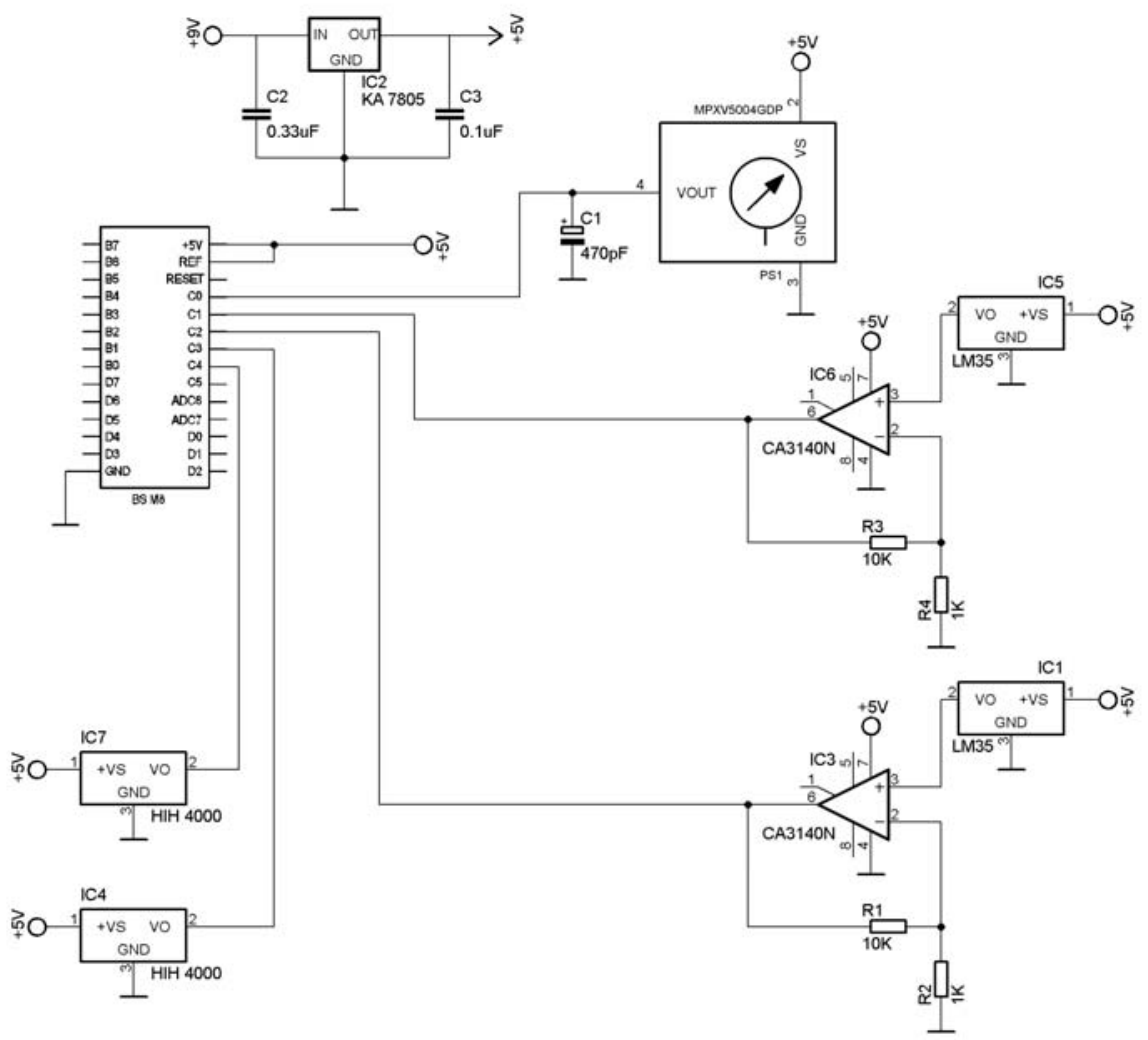

Figura 3. Diagrama esquemático do circuito eletrônico do sistema de aquisição de dados. 
(a)

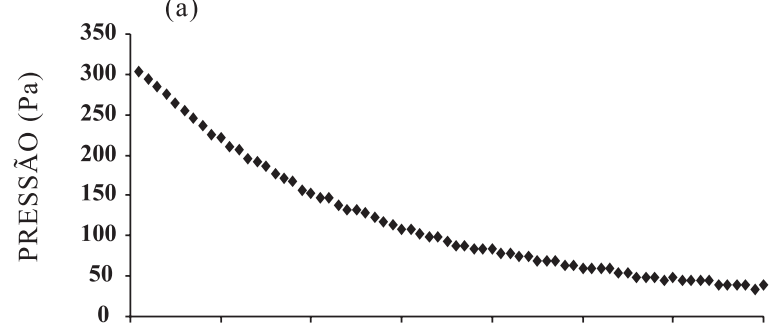

(b)

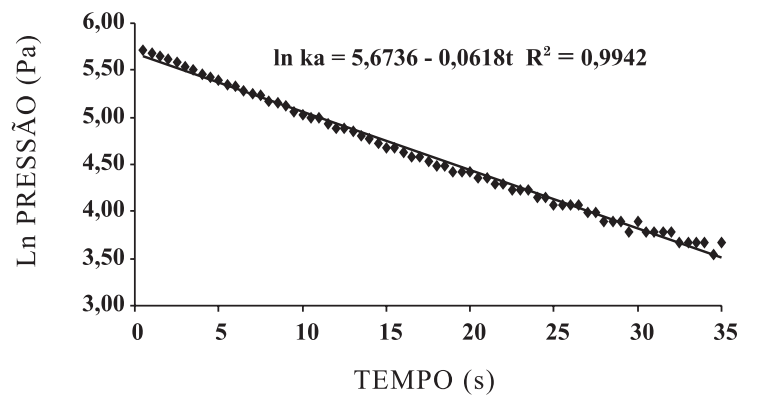

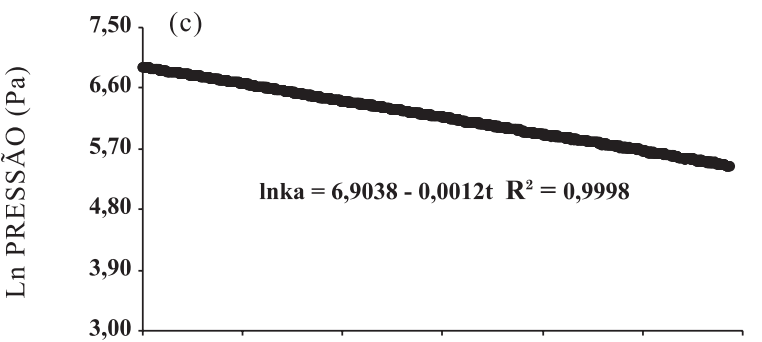

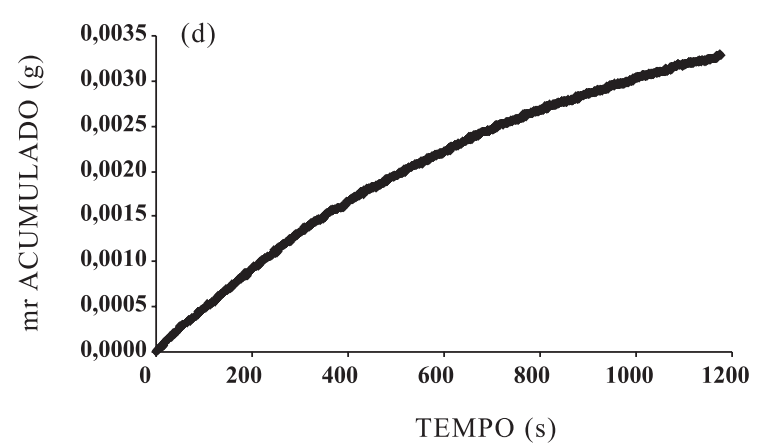

Figura 4. Os gráficos (a) e (b) correspondem à medida da pressão em função do tempo para os valores originais e transformados em logaritmo natural. Os gráficos (c) e (d) correspondem ao logaritmo natural da pressão e massa de água removida da amostra (Latossolo Vermelho) em função do tempo, respectivamente.

ar em função da temperatura média, ao longo do processo de medida, o intervalo de confiança da permeabilidade foi de $9,42 \pm 0,085 \mu \mathrm{m}^{2}$.

Com a análise estatística exploratória, verificouse que os dados possuem distribuição normal de probabilidade (Figura 5a), testado por Shapiro-Wilk $(\mathrm{W}=0,98$ e valor- $\mathrm{p}=0,246)$. O teste $\mathrm{F}$ foi significativo (valor $\mathrm{p}=0.0327$ ), o que significa que as médias dos dois tratamentos são significativamente diferentes. Mesmo assim, procedeu-se ao teste de médias, utilizando o teste $\mathrm{t}(0,05$ de probabilidade), revelando a influência da temperatura na medição da permeabilidade do solo ao ar (Figura 5b).

Como a viscosidade do ar depende da temperatura, a utilização do seu valor na equação (3), considerando a temperatura média durante o processo de determinação da permeabilidade, pode remover parte dos efeitos externos ao equipamento e à amostra de solo. Essa melhoria na estimativa da permeabilidade deu-se numa faixa de variação de 20,36 a $22,54{ }^{\circ} \mathrm{C}$, considerada pequena, o que demonstra a importância de utilizar esse procedimento, principalmente em medidas feitas ao longo do dia e quando a oscilação térmica for intensa. Mudanças na temperatura causam variações opostas na viscosidade de gases e líquidos: diminuição na temperatura causa diminuição na viscosidade de um gás, enquanto para um líquido há aumento, com exceção para gases em altas pressões, em que aumento de temperatura provoca diminuição da viscosidade (Bear, 1972; Libardi, 2005). Dessa maneira, quanto maior a temperatura, maior é o valor da viscosidade dinâmica do ar (Figura 6a) e, consequentemente, menor será a estimativa da permeabilidade do solo ao ar, resultando numa
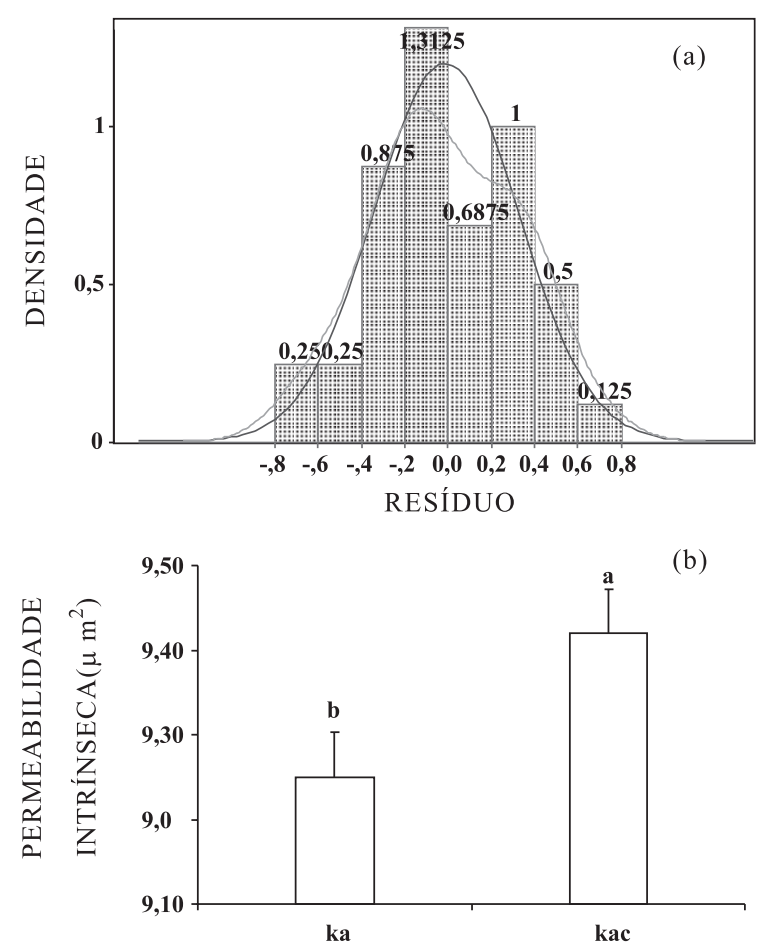

Figura 5. Histograma de distribuição de densidade de probabilidade dos dados (curva cinza) e de uma distribuição normal (a) e permeabilidades do solo ao ar sem (ka) e com (kac) a correção da viscosidade dinâmica do ar em função da temperatura. Médias comparadas pelo teste $t$ $(0,05$ p.) (b).

correlação negativa entre a permeabilidade e a temperatura (Figura 6b). Essa relação pode ser 


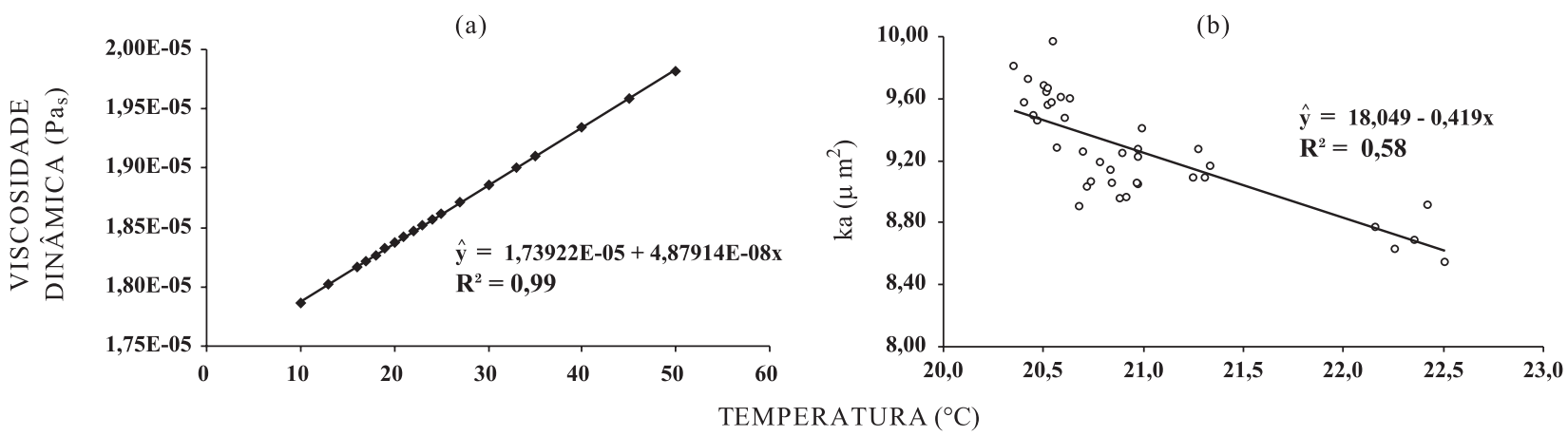

Figura 6. Regressão linear da viscosidade dinâmica do ar (a) e da permeabilidade intrínseca do solo ao ar (b) em função da temperatura ambiente. Os pares de dados apresentados em A foram obtidos no sítio (http://www.lmnoeng.com/Flow/GasViscosity.htm).

observada pela própria equação de Darcy-Buckingham, na qual a condutividade é diretamente proporcional à permeabilidade intrínseca e inversamente proporcional à viscosidade do fluido, e também pela própria equação da permeabilidade intrínseca (3), ressaltando que o modelo assume o valor modular do coeficiente angular da regressão linear da diminuição da pressão manométrica em função do tempo.

A medição realizada com a amostra do Latossolo Vermelho Amarelo foi tão precisa quanto a da amostra-padrão, e a estimativa da remoção de água da $\operatorname{amostra}\left(m_{r}\right)$ foi de 3,279 $\mathrm{mg}$ (Figura 4c,d). Levandose em conta que a diferença de conteúdo de água de uma amostra de solo entre potenciais mátricos variando de 1 em $1 \mathrm{kPa}$ até - $10 \mathrm{kPa}$ é de aproximadamente 1,5 g (para um solo de textura média), essa perda de água não é considerada alta e não deve alterar significativamente a estimativa da permeabilidade. Entretanto, é possível que, mesmo para solos com textura média, mas principalmente com textura mais argilosa, e para estimativas de permeabilidade ao ar feitas em potenciais mátricos próximos à saturação, a remoção de água cause modificações significativas nessa estimativa, uma vez que a duração da análise é maior.

O sistema automático de aquisição de dados funcionou adequadamente, possibilitando uma estimativa precisa da permeabilidade ao ar. A utilização do conversor A/D de 10 bits, do microcontrolador embarcado BS-M8, do regulador de voltagem e da amplificação de sinal para os sensores de temperatura foi satisfatória. A temporização feita via programa, utilizando-se de um recurso do Delphi, mostrou atraso de cerca de 1 s a cada 10 min, aproximadamente. Contudo, isso não alterou o desempenho das leituras.

\section{CONCLUSÕES}

1. O sistema automático de aquisição de dados para determinação da permeabilidade intrínseca do solo ao ar apresentou alto nível de precisão.
2. A medida da temperatura durante o processo de estimativa da permeabilidade é essencial para determinação da permeabilidade, uma vez que há modificação significativa na viscosidade dinâmica do ar.

3. A estimativa de remoção de água da amostra de solo estudado não foi significativa, porém, para outras condições de estudo é possível que haja alteração na determinação da permeabilidade ao ar.

\section{AGRADECIMENTOS}

Os autores agradecem a colaboração do Professor Tarlei Arriel Botrel (Departamento de Engenharia de Biossistemas da ESALQ/USP) e ao Engenheiro Eletrônico Márcio José Soares (www.arnerobotics.com.br).

\section{LITERATURA CITADA}

BEAR, J. Dynamics of fluids in porous media. New York, Elsevier, 1972. 764p.

BLACKWELL, P.S.; RINGROSE-VOASE, A.J.; JAYAWARDANE, N.S.; OLSSON, K.A.; MCKENZIE, D.C. \& MASON, W.K. The use of air-filled porosity and intrinsic permeability to air to characterize structure of macropore space and satured hydraulic conductivity of clay soils. Soil Sci., 41:215228,1990

BORLAND. Delphi 7 Personal. 2002. Disponível em: <http:// www.borland.pl/downloads> Acesso em: 2007.

CAVALIERI, K.M.V.; SILVA, A.P.; TORMENA, C.A.; LEÃO, T.P.; DEXTER, A.R. \& HÅKANSSON, I. Long-term effects of no-tillage on dynamic soil physical properties in a Rhodic Ferrasol in Paraná, Brazil. Soil Tillage Res., 103:158-164, 2009.

CRNILA, D. 2005 [online]. ComPort Library version 3.10. Disponível em: <http://sourceforge.net/projects/comport> Acesso em: 2009. 
FAHRAN, S; HOLSEN, T.M. \& BUDIMAN, J. Interaction of soil air permeability and soil vapor extraction. J. Environ. Eng., 127:32-37, 2001.

FISH, A.N. \& KOPPI, A.J. The use of a simple field air permeameter as a rapid indicator of functional soil pore space. Geoderma, 63:255-264, 1994.

KIRKHAM, D. Field method for determination of air permeability of soil in its undisturbed state. Soil Sci. Soc. Am. Proc.,11:93-99, 1946.

IVERSEN, B.V.; SCHJONNING, P.; POULSEN, T.G. \& MOLDRUP, P. In situ, on-site and laboratory measurements of soil air permeability: Boundary conditions and measurement scale. Soil Sci., 166:97-106, 2001.

LIBARDI, P.L. Dinâmica da água no solo. São Paulo, Edusp, 2005. 335p.

LOLL, P.; MOLDRUP, P.; SCHJONNING, P. \& RILEY, H. Predicting saturated hydraulic conductivity from air permeability: Application in stochastic water infiltration modeling. Water Res. Res., 35:2387-2400, 1999.
MCKENZIE, B.M. \& DEXTER, A.R. Methods for studying the permeability of individual soil aggregates. J. Agric. Eng. Res., 65:23-28, 1996.

NEVES, C.M.N.; SILVA. A.P. \& LEÃO, T.P. Permeabilidade ao ar em amostras de solo pelo método de pressão decrescente. In: REUNIÃO BRASILEIRA DE MANEJO E CONSERVAÇÃO DO SOLO E ÁGUA, 15., Santa Maria, 2004. Resumos... Santa Maria, 2004. CD ROM.

POD’YACHEV, S.P. Sgraph version 2.4. Disponível em: http:/ /pod0.chat.ru Acesso em: 2001.

SILVA, A.P.; LEAO, T.P.; TORMENA, C.A. \& GONCALVES, A.C.A. Determination of air permeability in undisturbed soil samples by the decreasing pressure method. R. Bras. Ci. Solo, 33:1535-1545, 2009.

STEINBERNNER, E.C. A portable air permeameter for forest soils. Soil Sci. Soc. Am. J., 23:478-481, 1959.

TYNER, J.S.; WRIGHT, W.C.; LEE, J. \& CRENSHAW, A.D. A dynamic air permeameter for coarse-textured soil columns and cores. Vadose Zone J., 4:428-433, 2005.

van GENUCHTEN, M.T.A closed-form equation for predicting the hydraulic conductivity of unsaturated soils. Soil Sci. Soc. Am. J., 44:892-898, 1980. 